\title{
Faire progresser la médecine de famille pour la santé et une vie meilleure
}

\author{
Retour sur 2020
}

Francine Lemire MDCM CCMF FCMF CAÉ IAS.A, DIRECTRICE GÉNÉRALE ET CHEF DE LA DIRECTION

Eric J. Mang MPA, DIRECTEUR GÉNÉRAL, SERVICES AUX MEMBRES ET RELATIONS EXTERNES

Chers collègues,

Alors que l'année touche à sa fin, il est temps de réfléchir à nos importantes réalisations. Les jalons et activités à célébrer au sein du Collège sont nombreux, mais nous pensons surtout aux efforts de plaidoyer déployés pour valider et soutenir le travail des médecins de famille et leurs cabinets dans un contexte de société et de pratique changeant. Nous vous invitons à lire notre rapport annuel ${ }^{1}$.

En janvier, nous avons appuyé la Déclaration d'Astana², ce qui a permis à la médecine familiale de se prononcer en faveur de soins communautaires solides. En collaboration avec l'Association médicale canadienne (AMC) et le Collège royal, nous avons publié le rapport du Groupe de travail sur les soins virtuels en février ${ }^{3}$. Le travail des 4 sous-groupes (interopérabilité et gouvernance, permis d'exercice et qualité des soins, éducation et modes de rémunération) reste pertinent, surtout au vu de l'expérience importante que nous vivons avec la maladie à coronavirus 2019 (COVID-19). Le rapport souligne l'importance des soins virtuels à l'intérieur d'une relation préétablie avec le prestataire de soins principal, ainsi que de leur rémunération équitable. Nous avons tous subi les répercussions de la COVID-19 en mars; comme les médecins de famille, nous nous sommes mis en télétravail pour assurer la continuité des opérations. Nous avons offert des webinaires, des billets sur le blogue et des ressources à jour sur la COVID-19, et avons prolongé d'un an les exigences annuelles pour la déclaration de crédits Mainpro+. En avril, la Fondation pour l'avancement de la médecine familiale a reçu un don de 5 millions de $\$$ de la Fondation de l'AMC pour financer des demandes de subvention de médecins de famille. Nous avons reçu plus de 130 propositions décrivant les mesures que des prestataires de première ligne ont vite mises en œuvre pour continuer de soigner les Canadiens en temps de pandémie. Les résultats d'un sondage sur la COVID-19 du CMFC diffusé en mai ont mis en évidence la pluripotence des médecins de famille et de notre discipline ${ }^{4}$. Plus de $90 \%$ des répondants ont élargi leur champ d'activité ou leurs lieux de pratique pour répondre aux besoins durant la $1^{\text {re }}$ vague de la pandémie. Dans un communiqué de presse, le CMFC a souligné que les modes de paiement alternatifs ont permis une plus grande stabilité de la pratique et aidé à répondre aux besoins complexes des patients atteints de maladies chroniques ${ }^{5}$. En réponse à l'agitation sociale au sud de la frontière, à la reconnaissance du racisme systémique au Canada et au mouvement Black Lives Matter, le CMFC a publié en juin une déclaration contre le racisme ${ }^{6}$.

En juillet, nous avons diffusé un résumé à jour des données probantes pour la vision du Centre de médecine de famille ${ }^{7}$. Comme ce modèle de soins en équipe continue d'être étayé par de solides données probantes, nous continuons de promouvoir son adoption avec l'aide de nos sections provinciales. En août, nous avons accueilli $\mathrm{D}^{\text {re }}$ Sarah Funnell, une médecin de famille autochtone, au sein de notre Conseil d'administration. La direction du CMFC veille à ce que les recommandations de la Commission de vérité et réconciliation soient respectées et à ce que l'équité, la diversité et l'inclusion soient encouragées. Nous sommes plus forts ensemble lorsque nous célébrons la diversité. Nous avons été très choqués et attristés par la mort tragique de $\mathrm{D}^{\mathrm{r}}$ Walter Reynolds en Alberta.

Par l'entremise de $D^{\text {re }}$ Shirley Schipper, présidente sortante du CMFC, nous avons plaidé en faveur d'un retour à la table de négociation avec les médecins en Alberta. En septembre, le plus grand groupe de candidats à ce jour a passé notre examen de médecine d'urgence, et nous avons célébré les 35 ans du programme Autoapprentissage. Grâce au soutien financier de l'AMC en octobre, nous avons entamé une initiative de développement professionnel en ligne sur la résilience des médecins de famille et de leurs cabinets. Nous avons aussi accueilli plus de 3000 participants à notre tout premier Forum en médecine familiale virtuel en novembre.

$N^{\prime}$ oublions pas de mentionner le $25^{\mathrm{e}}$ anniversaire de la Section des chercheurs, une médaille d'or pour le MFC aux Prix du magazine canadien dans la catégorie Meilleure illustration et l'augmentation du facteur d'impact à 3,112; ainsi que nos progrès afin de protéger la terminologie de la médecine de famille.

Nous savons comme l'année 2020 a été difficile, professionnellement et personnellement, pour beaucoup d'entre vous. Alors qu'elle s'achève, nous espérons que vos collègues et vous-même prendrez le temps de faire une pause, de rester en contact avec votre famille et vos amis (peu importe de quelle façon) et de réfléchir à votre contribution positive dans la vie de vos patients et dans votre communauté au cours de cette année sans précédent.

En notre nom à tous au CMFC, nous vous souhaitons nos meilleurs vœux des Fêtes et pour 2021. 